This item was submitted to Loughborough's Research Repository by the author.

Items in Figshare are protected by copyright, with all rights reserved, unless otherwise indicated.

\title{
Twisting techniques used in dismounts from rings
}

PLEASE CITE THE PUBLISHED VERSION

PUBLISHER

(C) Human Kinetics

VERSION

NA (Not Applicable or Unknown)

LICENCE

CC BY-NC-ND 4.0

REPOSITORY RECORD

Yeadon, Maurice R.. 2019. "Twisting Techniques Used in Dismounts from Rings". figshare. https://hdl.handle.net/2134/8674. 
This item was submitted to Loughborough's Institutional Repository (https://dspace.lboro.ac.uk/) by the author and is made available under the following Creative Commons Licence conditions.

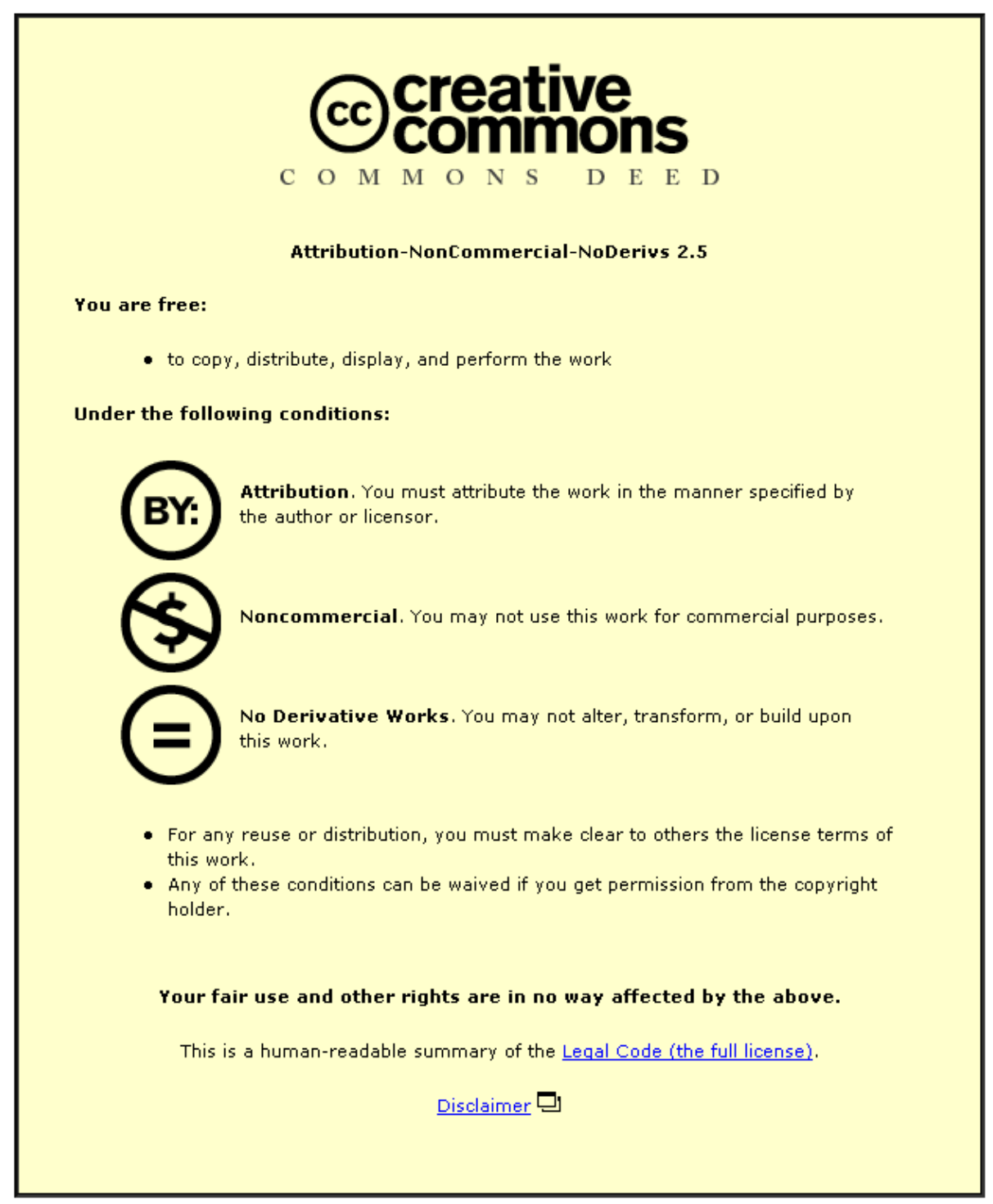

For the full text of this licence, please go to: http://creativecommons.org/licenses/by-nc-nd/2.5/ 


\title{
Twisting Techniques Used in Dismounts from the Rings
}

\author{
Maurice R. Yeadon \\ Sports Biomechanics Laboratory, Department of Sports Science, \\ Loughborough University, LE11 3TU, UK
}

\begin{abstract}
At the 1992 Olympic Games six full twisting double somersault dismounts were recorded with two video cameras during the rings individual apparatus finals in the men's Artistic Gymnastics competition. Angles describing body configuration were determined from video data and were input, together with initial orientation angle values and angular momentum components, into a computer simulation model of aerial movement. Mean absolute deviations between simulation and video after the completion of one half twist were $0.01 \mathrm{rev}$ for somersault, $2.8^{\circ}$ for tilt, and 0.08 rev for twist. When the estimate of the initial tilt angle was adjusted by up to $1^{\circ}$ these deviations fell to $1.6^{\circ}$ for tilt and $0.02 \mathrm{rev}$ for twist. All 6 competitors produced the majority of the tilt using aerial techniques that were predominantly asymmetrical movements of the arms. Contributions to the subsequent removal of tilt were determined using reverse simulations, and again arm movements were the main contributors.
\end{abstract}

\section{Introduction}

Dismounts from the rings comprise somersaults with or without twist. In non-twisting dismounts the gymnast must leave the apparatus with the appropriate linear and somersaulting velocities and must then adjust body configuration during flight to obtain the correct somersault angle at landing. In twisting dismounts, in addition to meeting these requirements, the gymnast must also initiate and control twisting to produce the required amount of twist prior to landing. Twisting techniques may be divided into those that start the twist during contact with the apparatus and those that initiate the twist during the aerial phase. This gives rise to the terms contact techniques and aerial techniques for the production of twist.

Al-Haroun (1980) filmed 6 gymnasts performing full twisting somersault dismounts from the rings. It was found that 3 gymnasts started the twist before releasing first one ring and then the other, while the remaining 3 released the two rings simultaneously and appeared to initiate the twist after release. Such cinematographical studies of twisting somersaults can establish the orientations of the body throughout a movement together with the relative configurations of body segments. This information may suggest that a particular type of twist (e.g., contact, aerial) was used, but without a quantitative mechanical analysis, the conclusions that can be drawn are limited.

If a gymnast has completed some of the twist and has a twisting velocity when the rings are released, it may seem reasonable to conclude that the twisting technique employed by the gymnast was one of starting the twist during the contact phase. This may not be the case. If a somersault without twist is analyzed, the amounts of twist and twisting velocity at takeoff will not be exactly zero and so it is a matter of the mechanical significance of these values. Even if the gymnast does have a substantial contribution from the contact phase, this contribution may not be the major one. Additionally, any actual performance will exhibit configurational changes that may be interpreted as showing that a particular technique contributes to the twist. Without some way of quantifying the contributions of each technique in a twisting somersault, any conclusion reached on the origin of the twist is likely to reflect the investigator's personal opinions.

A more conclusive result was obtained by Van Gheluwe (1981), who used a six-segment computer simulation model to evaluate filmed performances of three full twisting somersaults on a trampoline. By using simulations based on modifications of the film data, he was able to show that arm movements during the aerial phase were responsible for most of the twist. A method of partitioning twist contributions has been developed by Yeadon (1993d), who uses the angle of tilt as a measure of the twisting potential 
present. For full twisting somersault dismounts from the high bar, Yeadon, Lee, and Kerwin (1990) found that gymnasts used asymmetrical movements of the arms and hips to produce tilt and twist in the aerial phase. For double somersault high-bar dismounts with twist, it was found that contact contributions were larger when the twist occurred early in the first somersault (Yeadon, 1991).

Since twisting technique therefore appears to depend on the type of dismount, it will be productive to analyze the same dismount performed by a number of competitors. In this study the double backward somersault straight with a full twist in the second somersault was selected as the rings dismount to be analyzed.

\section{Methods}

\section{Data Collection}

Video recordings were made of the rings individual apparatus finals in the men's Artistic Gymnastics competition at the Olympic Games in Barcelona in July 1992. Two Panasonic MS2 SVHS cameras were located in the gantry of the Palau Sant Jordi Centre so that the camera axes made angles of approximately $45^{\circ}$ with the vertical plane of the rings frame. The field of view of each camera was approximately $10 \mathrm{~m}$ wide and included eight control points on the rings apparatus for camera calibration (Figure 1). Since the cameras recorded 50 fields per second, this resulted in around 50 fields for each dismount. The cameras were used with electronic $1 / 250 \mathrm{~s}$ shutters in order to give a sharp image of rapidly moving body segments. Measurements were taken prior to the competition with a flexible tape so that the three-dimensional locations of the eight control points on the rings apparatus could be determined. The vertical alignment of the rings frame was checked by sighting from the side and using the suspended rings as plumb bobs.
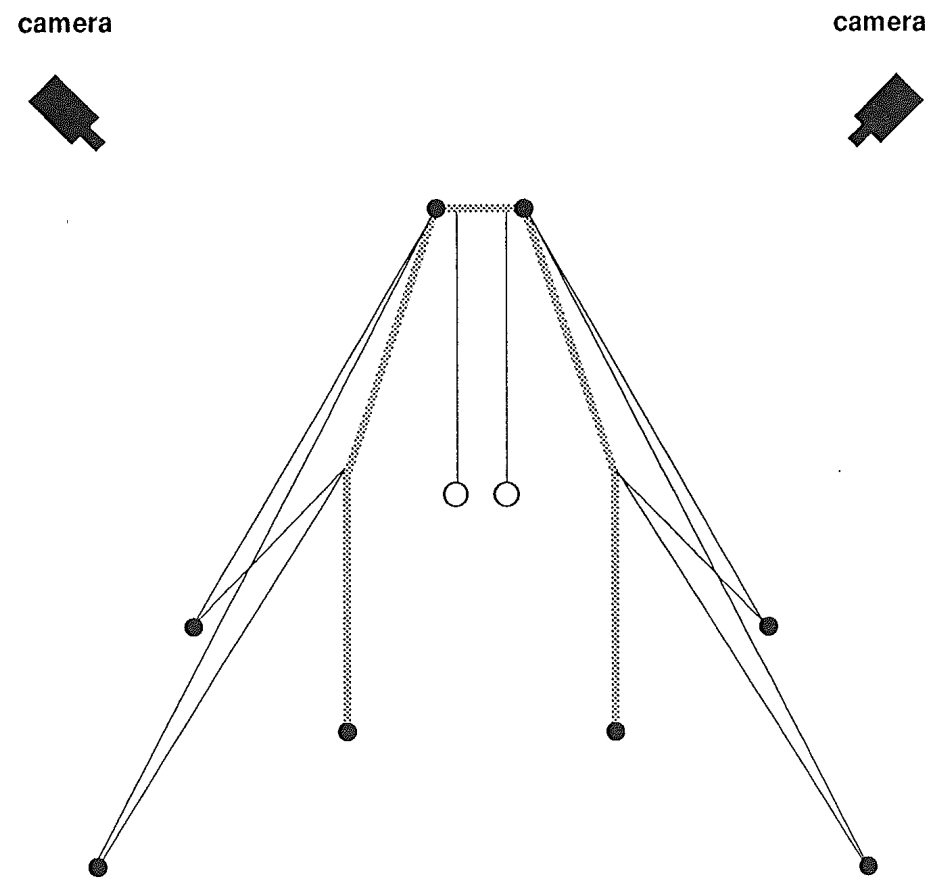

Figure 1: The eight control points on the rings apparatus.

\section{Data Analysis}

The most popular dismount used in the rings exercises of the team optionals and individual apparatus competitions was the double backward somersault straight with a full twist in the second somersault. Of 
the 8 gymnasts in the finals of the rings individual competition, 6 used this dismount. This resulted in competitors from Hungary and Bulgaria, with 2 competitors each from China and the Soviet States.

The two views of the aerial phase of each dismount were digitized with a Prisma 3 graphics image capture board and custom software for obtaining the data from cursor position using an Archimedes computer. The images of the eight control points were digitized in each of two fields prior to each gymnast mounting the apparatus, and the resulting 12 data sets were used to obtain mean coordinate values. From the measured three-dimensional locations of the eight control points on the rings apparatus, together with the digitized data, the 11 direct linear transformation (DLT) camera parameters were determined using the method of Abdel-Aziz and Karara (1971).

In each video field of the aerial phase of the dismount, the wrist, elbow, shoulder, hip, knee, and ankle centers were digitized, giving 12 landmarks in total. The head was assumed to be in normal alignment and the ankles were assumed to be plantar flexed. Error estimates of the coordinate data were obtained by comparing each value with the mean of the corresponding values from adjacent video fields. A combination of global and local error estimates was then used as described in Yeadon (1990a) for curvefitting the coordinate data using the quintic spline of Wood and Jennings (1979). These mathematical fits to the digitized data permitted the estimation of data values at times in between those of the video fields.

The camera positions were back-calculated from the DLT coordinates, and the digitized data sets from the two cameras were synchronized by determining rays from each camera to an unweighted mean position of the 12 digitized points for the last three fields of each dismount from each camera. The method described in Yeadon (1989) was used to determine the time of the penultimate field from one camera in terms of the time scale of the other camera. The quintic spline fits to the displacement data were then used to obtain interpolated digitized values to produce synchronous data sets.

The body was modeled with four arm segments, four leg segments, and three torso segments. The orientation of the body was defined by angles corresponding to somersault, tilt, and twist, where somersault is the rotation about an axis parallel to the angular momentum vector, twist is the rotation about the longitudinal body axis corresponding to minimum moment of inertia, and tilt is the angle between the longitudinal axis and the plane perpendicular to the angular momentum vector (Figure 2). Body configuration was specified with 14 angles to describe the relative orientations of the body segments arising from movement at the elbows, shoulders, hips, knees, and two points on the spine (Yeadon, 1990c). The three-dimensional coordinates of the digitized joint centers were reconstructed from the digitized data, and the time histories of the orientation and configuration angles were determined (Yeadon, 1990a).

Segmental inertia parameters for each gymnast were determined with the model of Yeadon (1990b). Since this model is based upon 95 anthropometric measurements that were not available for each gymnast, the following procedure was adopted. The height and weight of each gymnast were obtained from the biographical files available for the media via touch-screen computer in Barcelona. Segment lengths were calculated from the three-dimensional locations of the 12 digitized body landmarks during the dismount. The remaining anthropometric length measurements needed were estimated from these segment lengths using regression equations as described in Yeadon, Challis, and Ng (1993). Perimeter and width measurements were estimated using regression equations based upon height and weight. An 11-segment model was used to determine the angular momentum vector during each dismount using the time histories of the 17 orientation and configuration angles (Yeadon, 1990c). The mean values of the calculated angular momentum components and the time histories of the 14 configurational angles, together with the initial values of the three orientation angles and the segmental inertia parameters, were used as input to an 11-segment simulation model (Yeadon, Atha \& Hales, 1990).

The relationship between the twist rate p, the tilt angle $\theta$ and the somersault rate $\Omega$ for an axially symmetric rigid body with principal moments of inertia $A$ and $A$, and $C(A>C)$ is given in Yeadon (1993a) as:

$$
p=\Omega(A / C-1) \sin \theta
$$

Thus the angle of tilt between the longitudinal axis of the body and the invariable plane perpendicular to the angular momentum vector may be used to give the number of twists per somersault for a given body configuration, providing that the two large principal moments of inertia are approximately equal. Since the angle of tilt is approximately proportional to the twist rate, it may therefore be used as a measure of the twisting potential present, independent of the actual configuration adopted. Twisting 


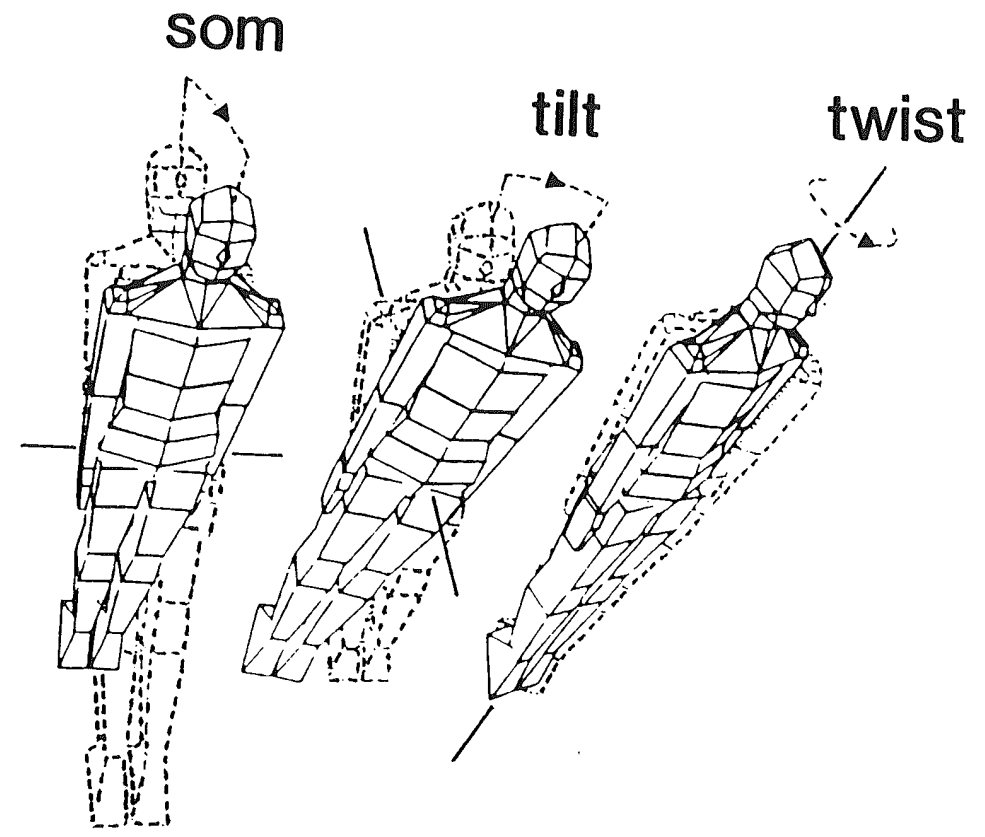

Figure 2: Angles of somersault, tilt, and twist.

techniques may be defined as contact or aerial techniques depending on whether the twist is initiated during contact with the rings apparatus or during the aerial phase (Yeadon, 1993b, 1993c). The initial value of the tilt angle when the rings are released is a measure of the twist initiated during contact with the rings. The subsequent increase in the tilt angle during the dismount gives the contribution of aerial twisting techniques to the total tilt produced.

In order to partition the aerial contribution to the tilt angle into a symmetry contribution and contributions associated with asymmetries of arms, chest, and hips, the configurational angles obtained from video were modified. These modifications comprised the systematic removal of asymmetries of the arms, chest, and hips about the sagittal plane. Arm movement was made symmetrical by having the left arm mirror the recorded movement of the right arm and vice versa; chest torsion relative to the thorax was eliminated; and hip movement was restricted by only permitting flexion parallel to the sagittal plane.

The systematic removal of arm, chest, and hip asymmetries provided 12 configuration sequences ranging from the original movement to a sequence with complete left-right configurational symmetry. The tilt angle produced at the time corresponding to one half twist in the original movement was determined from simulations based upon the modified configurations. Since arm asymmetries were removed in two ways, the tilt values were averaged in these simulations to give a total of eight tilt values for the modified simulations. The tilt contribution due to symmetry was calculated as the amount that the tilt angle in the simulation, in which left-right symmetry was maintained, rose above the initial value.

The tilt associated with asymmetrical arm movement could be calculated as the difference between the tilt values for the simulations of complete symmetry and of symmetrical chest and hips. It could also be calculated as the difference between the tilt values for the simulations corresponding to arm symmetry and the original configuration. The average of the tilt values obtained in these two ways was taken as the tilt contribution from asymmetrical arm movement. The other contributions to the tilt angle were calculated in a similar way as described in Yeadon (1993d).

In order to determine the techniques used for the removal of tilt and the stopping of the twist, the landing of the dismount was used as a starting point and simulations were run backward in time. The same procedure for partitioning the contributions to the production of tilt was used to determine the contributions to the removal of the tilt present at the half twist position. 


\section{Results}

Since the interpolated information sets from the two cameras were synchronized using the displacement data, the accuracy of the synchronization depends upon the accuracy of the displacement data. As the data on the finals of the individual rings competition were obtained from a single continuous video recording, a measure of the synchronization error was obtained by comparing the six time offsets with their mean value. The mean deviation from this value was $0.002 \mathrm{~s}$. This should be regarded as an underestimate of the synchronization error since there will also be some systematic error associated with the calculated camera positions.

An estimate of the accuracy of the DLT reconstruction was obtained by comparing the calculated coordinates of the eight control points with their measured locations. The coordinate differences had an unbiased root mean square value of $0.017 \mathrm{~m}$ with a maximum deviation of $0.034 \mathrm{~m}$. The mean of the error estimates of the digitized coordinate values obtained from a comparison of a data value with the mean of the corresponding values from adjacent fields was $0.017 \mathrm{~m}$ for the $10 \mathrm{~m}$ field of view. The mean of the error estimates of the orientation and configuration angles was $1.5^{\circ}$.

The differences between the values of the orientation angles obtained from simulation and video were evaluated at the time for which the video value of the twist angle was 0.5 revolutions. Mean deviations were $0.01 \mathrm{rev}$ for somersault, $2.8^{\circ}$ for tilt, and $0.08 \mathrm{rev}$ for twist. When the value of the initial tilt angle was allowed to be adjusted by up to $1^{\circ}$ to improve the agreement between the twist values, these deviations fell to $1.6^{\circ}$ for tilt and 0.02 rev for twist. For reverse simulations the deviations between simulation and video at the half twist position were $0.01 \mathrm{rev}$ for somersault, $2.2^{\circ}$ for tilt, and $0.06 \mathrm{rev}$ for twist. When the value of the final tilt angle was allowed to be adjusted by up to $1^{\circ}$, the deviations became $2.3^{\circ}$ for tilt and $0.03 \mathrm{rev}$ for twist. Figure 3 presents a comparison of video and simulation sequences for Gymnast 06 for a forward simulation.

Tilt contributions for the production of twist were evaluated for the time at which the original simulation reached a twist value of one half revolution. This twist value was chosen since it occurred approximately at the midflight time and corresponded to the maximum tilt value. Table 1 presents the contributions made to the tilt angle at the half twist position in each of the six dismounts.
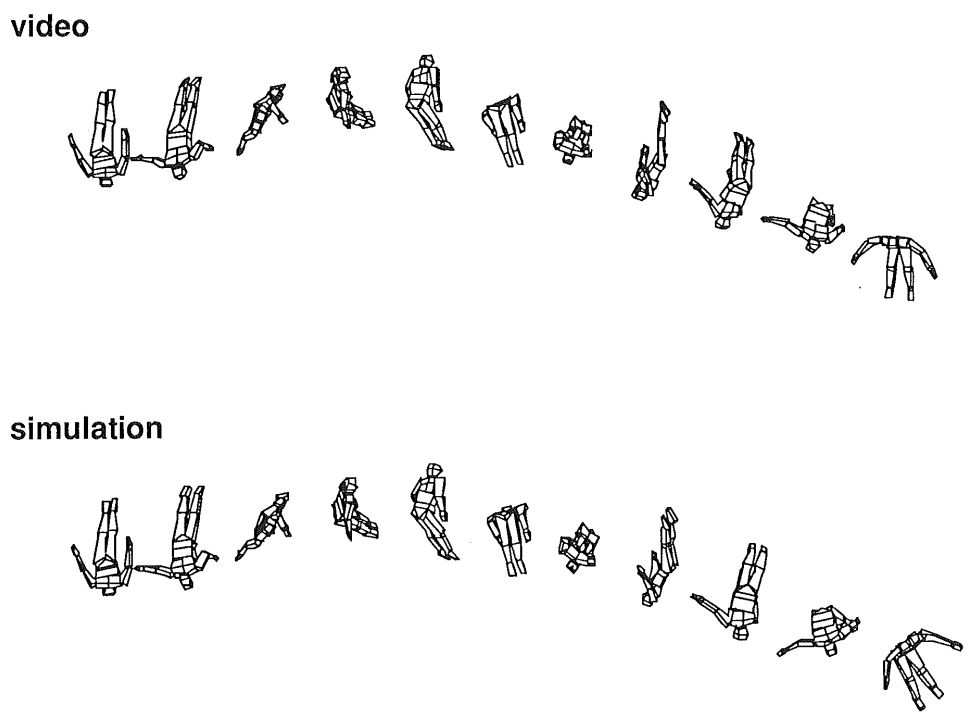

Figure 3: Comparison of video and simulation for Gymnast 06.

The contact contribution is the initial tilt angle between the longitudinal axis and the invariable plane perpendicular to the angular momentum vector evaluated at the moment of release. The symmetry contribution indicates how much the tilt angle increases from its initial value during a simulation that enforces symmetry of the arms, chest, and hips about the sagittal plane. The contributions listed under the headings Arms, Chest, and Hips arise from asymmetrical arm movements, torsion of the chest, and lateral flexion at the hips. 
Table 1: Contributions to the Production of Tilt (in degrees)

\begin{tabular}{lrrrrrrr}
\hline \multirow{2}{*}{ Gymnast } & Contact & Symmetry & Arms & Chest & Hips & Total & Actual \\
& & & & & & & \\
\hline 06 & 2 & -2 & 8 & 1 & 1 & 10 & 11 \\
17 & -3 & 10 & 11 & -7 & -1 & 9 & 9 \\
18 & -1 & -5 & 8 & -1 & 2 & 5 & 7 \\
22 & -1 & 0 & 13 & -3 & 0 & 9 & 9 \\
24 & 2 & 5 & 0 & -3 & 4 & 8 & 8 \\
47 & 0 & 2 & 2 & 2 & 3 & 10 & 9 \\
Mean & 0 & 2 & 7 & -2 & 2 & 8 & 9 \\
\hline
\end{tabular}

Note. Contributions have been rounded to the nearest degree.

Table 2: Contributions to the Removal of Tilt (in degrees)

\begin{tabular}{|c|c|c|c|c|c|c|c|}
\hline Gymnast & Final & Symmetry & Arms & Chest & Hips & Total & Actual \\
\hline 06 & 1 & 1 & 2 & 4 & 3 & 11 & 12 \\
\hline 17 & 0 & 5 & 2 & 1 & 0 & 7 & 8 \\
\hline 18 & 0 & 3 & 3 & 1 & 2 & 8 & 7 \\
\hline 22 & -1 & 6 & 3 & 0 & 0 & 8 & 8 \\
\hline 24 & 0 & 2 & 8 & 0 & 1 & 10 & 10 \\
\hline 47 & 0 & 4 & 3 & 2 & 1 & 8 & 8 \\
\hline Mean & 0 & 3 & 3 & 1 & 1 & 9 & 9 \\
\hline
\end{tabular}

Note. Contributions have been rounded to the nearest degree.

For each of the six dismounts analyzed, the deviations between the tilt contributions (as listed under Total in Table 1) and the value obtained from the unmodified simulation had a mean value of $0.8^{\circ}$ and a maximum of $1.9^{\circ}$. The values in Table 1 have been rounded to the nearest degree so that the value under the Total heading is not always exactly the same as the sum of the stated individual values.

Contributions for the removal of tilt were evaluated from the time at which the original reverse simulation reached a twist value of one half revolution. Table 2 presents the contributions made to the removal of tilt in each of the six dismounts. The Final contribution is the remaining tilt angle between the longitudinal axis and the invariable plane perpendicular to the angular momentum vector at the moment of landing. The Symmetry contribution indicates how much the tilt angle increases from its final value during a reverse simulation that enforces symmetry of the arms, chest, and hips about the sagittal plane. The contributions listed under Arms, Chest, and Hips are obtained in a similar way. For each of the six dismounts analyzed, the deviations between the tilt removal contributions (as listed under Total) and the value obtained from the unmodified simulation had a mean value of $0.4^{\circ}$ and a maximum of $0.9^{\circ}$.

\section{Discussion}

Table 1 shows that none of the gymnasts had substantial contact contributions to the production of tilt. Gymnasts 17 and 24 had symmetry contributions of $10^{\circ}$ and $5^{\circ}$, respectively, while the other four 
competitors had small or negative symmetry contributions. Asymmetrical arm movement was the major contributor, accounting by itself for the production of tilt in four cases. Chest contributions were all small or negative with only Gymnast 24 producing a substantial hip contribution of $4^{\circ}$.

Since the twist starts some time after takeoff it might be expected that contact and symmetry contributions would be small. However, Yeadon (1993e) showed that small initial tilt values can be used to produce late twisting movements by using a symmetrical wide arm configuration to accentuate the gradual buildup of tilt known as nutation. While their symmetry contributions indicate that Gymnasts 17 and 24 made some use of such a technique, the remaining four used other techniques. At release all gymnasts were slightly piked rather than arched. Yeadon (1993c) showed that asymmetries of the chest and hips are less effective for producing tilt from piked positions in backward rather than forward rotating somersaults. This may explain why many of the chest and hip contributions were small and even negative.

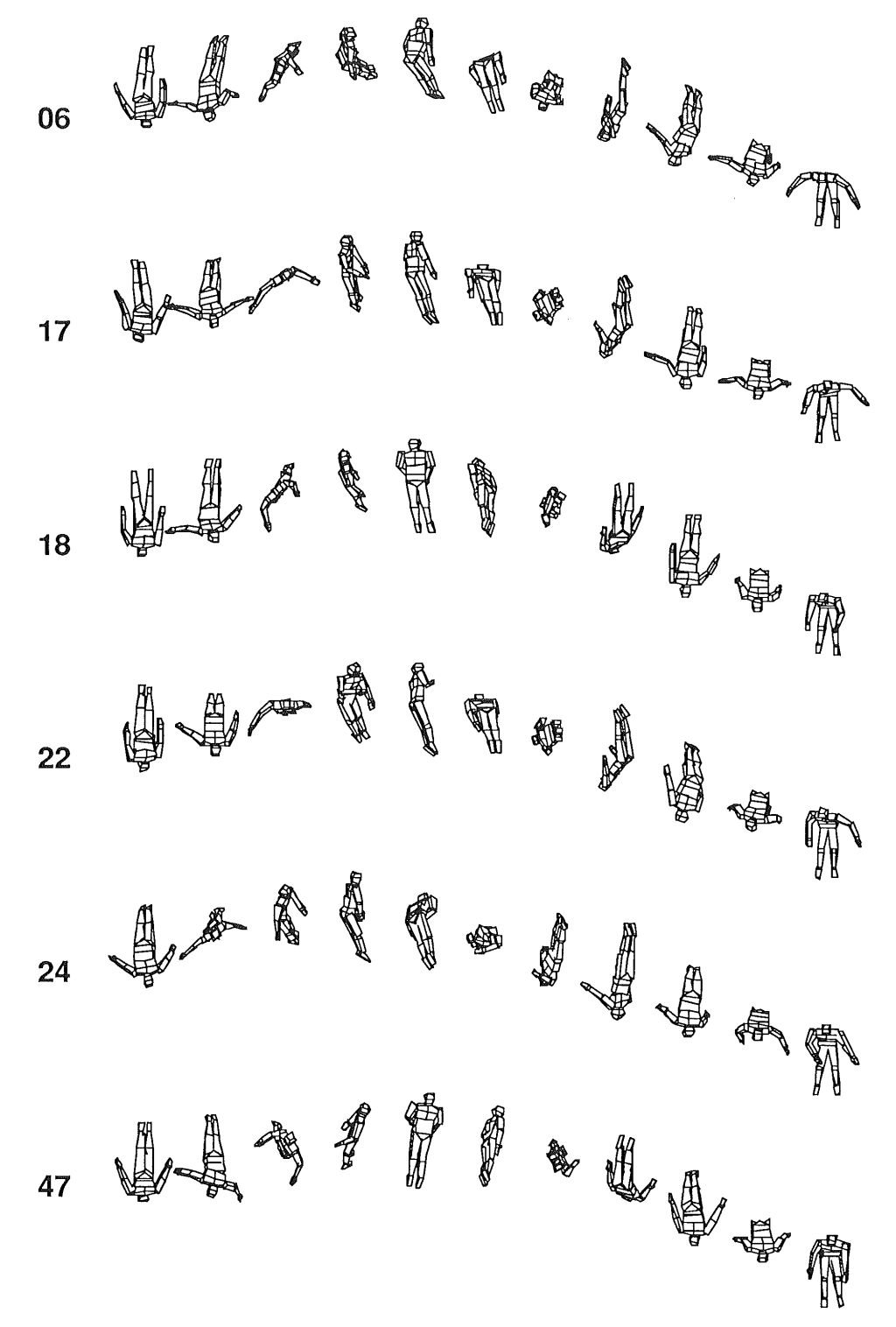

Figure 4: Graphics sequences of the six dismounts performed.

Although the major contributions have been identified as arm asymmetries, all gymnasts used arm configurations that were approximately symmetrical and all abducted both arms soon after release (Figure 4). A small arm asymmetry is sufficient to establish the direction of twist, and a wide arm configura- 
tion will accentuate the buildup of tilt as described in Yeadon (1993e). The movement may be coached using the following key points. Soon after takeoff both arms are abducted slightly asymmetrically: for a twist to the left, the left arm should be closer to the body than the right. The arms should remain wide until the quarter twist position is reached. At this point the tilt angle starts to fall, and so the arms should be adducted to minimize this effect and to accelerate the twist. Table 2 shows that all gymnasts had a final tilt angle very close to zero. This is important for stopping the twist and for achieving correct body alignment at landing. Symmetrical movements made substantial contributions to the removal of tilt, indicating that piking prior to landing automatically helped to reduce the angle of tilt. Arm asymmetries also made substantial contributions towards the removal of tilt. Although chest and hip contributions were generally small, it is interesting to note that all were positive. This fits in with the result of Yeadon (1993c) that chest and hip contributions are more effective in removing tilt than in producing tilt from a piked position in backward somersaults. The key points for coaching the removal of tilt are to pike prior to landing and to abduct the left arm if the twist is to the left.

\section{References}

Abdel-Aziz, Y.I., $\&$ Karara. H.M. (1971). Direct linear transformation from comparator coordinates into object space coordinates in close-range photogrammetry (ASP Symposium on Close-Range Photogrammetry). Falls Church, VA: American Society of Photogrammetry.

Al-Haroun, M.R. (1980). Three-dimensional cinematographic analysis of selected full twisting movements in gymnastics. Unpublished doctoral dissertation, Indiana University. Bloomington.

Van Gheluwe, B. (1981). A biomechanical simulation model for airborne twist in backward somersaults. Journal of Human Movement Studies, 7, 1-22.

Wood, G.A., \& Jennings, L.S. (1979). On the use of spline functions for data smoothing. Journal of Biomechanics, 12, 477-479.

Yeadon, M.R. (1989). A method for obtaining three-dimensional data on ski jumping using pan and tilt cameras. International Journal of Sport Biomechanics, 5, 238- 247.

Yeadon, M.R. (1990a). The simulation of aerial movement - I: The determination of orientation angles from film data. Journal of Biomechanics, 23, 59-66.

Yeadon, M.R. (1990b). The simulation of aerial movement - II: A mathematical inertia model of the human body. Journal of Biomechanics, 23, 67-74.

Yeadon. M.R. (1990c). The simulation of aerial movement - III: The determination of the angular momentum of the human body. Journal of Biomechanics, 23, 75-83.

Yeadon, M.R. (1991). Twisting double somersault dismounts from high bar. In R.N. Marshall \& L.S. Jennings (Eds.), Abstracts: Third International Symposium on Computer Simulation in Biomechanics (pp. 16-17). Perth: University of Western Australia.

Yeadon, M.R. (1993a). The biomechanics of twisting somersaults. Part I: Rigid body motions. Journal of Sports Sciences, 11, 187-198.

Yeadon, M.R. (1993b). The biomechanics of twisting somersaults. Part 11: Contact twist. Journal of Sports Sciences, 11. 199-208.

Yeadon, M.R. (1993c). The biomechanics of twisting somersaults. Part III: Aerial twist. Journal of Sports Sciences, 11, 209-218. 
Yeadon, M.R. (1993d). The biomechanics of twisting somersaults. Part IV: Partitioning performance using the till angle. Journal of Sports Sciences, 11, 219-225.

Yeadon, M.R. (1993e). Utilising nutation in twisting somersaults. In B. Landjerit (Ed.), Proceedings of the Fourth International Symposium on Computer Simulation in Biomechanics BMS3-16 (p. 17). Paris: Ecole Nationale Superiere d'Arts et Metiers.

Yeadon, M.R., Atha, J., \& Hales, F.D. (1990). The simulation of aerial movement - IV: A computer simulation model. Journal of Biomechanics, 23, 85-89.

Yeadon, M.R., Challis, J.H., \& Ng, R. (1993). Personalised segmental inertia parameters. In S. Bouisset, S. Metral \& H. Monod (Eds.), Abstracts of the International Society of Biomechanics XlVth Congress (pp. 1494-1495). Paris: Societe de Biomechanique.

Yeadon, M.R., Lee, S., \& Kerwin, D.G. (1990). Twisting techniques used in high bar dismounts. International Journal of Sport Biomechanics, 6, 139-146.

\section{Acknowledgments}

This research project was made possible by the support of the IOC Medical Commission, the Centre d'Alt Rendiment at Barcelona, and Loughborough University. 